Research Paper

\title{
Computer Simulation of Diffusion Potentials for Aqueous and Fused Salt Systems
}

\author{
Masatoshi OKADA and Kazutaka KaWAMURA \\ National Research Institute for Metals, \\ 2-3-12, Nakameguro, Meguro-ku, Tokyo
}

(Received June 2, 1970)

\begin{abstract}
The transition state of the diffusion potential for a free diffusion was simulated with a digital computer by using one-dimentional phenomenological equations. The simulation was performed for a fused salt junction; $\mathrm{AgNO}_{8}\left(0.5 \mathrm{~mol}\right.$ fraction), $\mathrm{KNO}_{8} / \mathrm{AgNO}_{8}(0.3), \mathrm{KNO}_{8}$ at $300^{\circ} \mathrm{C}$, and for an aqueous junction; $\mathrm{NaCl}(0.05 \mathrm{M}) / \mathrm{NaCl}(0.1)$ at $25^{\circ} \mathrm{C}$. The results are as follows: The time dependence of the diffusion potential is approximated to $V=V_{\infty}(1-\exp (-t / t))$. The time constants $\tau^{\prime} \mathrm{s}$ are $1.2 \times 10^{-18} \mathrm{sec}$ for the fused salt and $7.5 \times 10^{-10} \mathrm{sec}$ for the aqueous system. The length of time constant depends considerably on the dielectric constant of the solution. The diffusion proceeds much more rapidly in the aqueous junction than in the fused salt. The potential in the aqueous diffusion layer has its minimum near the contact plane.
\end{abstract}

\section{Introduction}

In an electrolyte solution, when diffusion coefficients of the ions of opposit charge are not equal, a simple diffusion across the liquid junction leads to continued separation of charge. However, on the other hand, migration in the collective field due to the separated charges counteracts further separation of charge, and ultimately the ions of opposit charge would diffuse each other at the same velocity as if a neutral species of the electrolyte diffuse under a certain electric field (diffusion potential). In this sense, the diffusion of an electrolyte in a solution as a model can be considered rather as a kind of electrochemical transference than as a pure diffusion.

The steady state value of the diffusion potential is theoretically calculable by the use of reversible or irreversible thermodynamics. In either case, however, the assumption of electroneutrality is a priori used in every part of the junction. This assumption is selfcontradictory because there can be no potential difference if there is no separation of charge as mentioned above. A precise approach to this problem may be made without self-contradiction if the condition of electroneutrality is replaced by that of Poisson's equation which gives the electrostatic field due to the separated charge.

The purpose of this work is to solve simultaneously the phenomenological equations about the constituent ions and Poisson's equation by the help of a digital computer. By the calculation about a fused salt and aqueous systems, we can understand the difference between the two systems.

\section{Experimental}

\subsection{The systems studied}

The calculations will be performed for the diffusion potentials across the following junctions.

(1) Fused salt system

$$
\text { (-) } \mathrm{AgNO}_{3}\left(0.5 \mathrm{~mol} \text { fraction), } \mathrm{KNO}_{3} / / \mathrm{AgNO}_{3}\right.
$$
$(0.3), \mathrm{KNO}_{3}(+)$ at $300^{\circ} \mathrm{C}$

The experimental diffusion potential of the above junction has been measured by the present authors with an observed value of $5.18 \mathrm{mV}^{1}$ ).

(2) Aqueous system

$$
\begin{aligned}
& (-) \mathrm{NaCl}(0.05 M), \mathrm{Aq} / / \mathrm{NaCl}(0.1 M), \\
& \mathrm{Aq} \text { at } 25^{\circ} \mathrm{C}
\end{aligned}
$$

\subsection{Diffusion model}

We will treat here one-dimentional free diffusion along the $X$ axis after allowing the contact of the two solutions at $X=0$. In the fused salt the external frame was chosen for reference of the migrational or diffusional velocity of ion, and in the aqueous solution a solvent water was chosen. As shown in Fig. 1, the part of the junction $(-\delta \leqslant X \leqslant+\delta)$ was separated at 


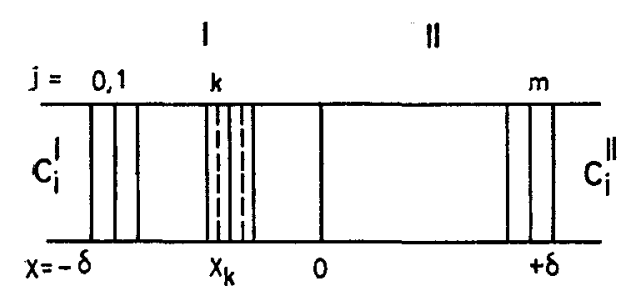

Fig. 1 Digital model of a free diffusion

fixed intervals by planes vertical to $\mathrm{X}$ axis. The cells are numbered in spacial sequence from 0 to $\mathrm{m}$.

\subsection{Equations}

In this work, it is assumed that the flux of the species $i$ depends only on the gradient of the electrochemical potential of $i$ itself at the point in question but not on those of any other ions. The equation for flux is thus

$$
\begin{gathered}
J_{\mathbf{i}}=-D_{\mathrm{i}}\left(\partial C_{\mathrm{i}} / \partial X\right)-C_{\mathrm{i}} D_{\mathrm{i}}\left(\partial \ln Y_{\mathbf{i}} / \partial X\right) \\
+C_{\mathrm{i}} U_{\mathrm{i}} F(\partial \phi / \partial X) \\
\mathrm{i}=\mathrm{Na}^{+}, \mathrm{Cl}^{-} \quad(\mathrm{a}) \\
\mathrm{i}=\mathrm{Ag}^{+}, \mathrm{K}^{+} \quad \text { (f) } \\
J_{\mathrm{k}}=C_{\mathbf{k}} U_{\mathrm{k}} F(\partial \phi / \partial X) \\
\mathrm{k}=\mathrm{NO}_{3}^{-}
\end{gathered}
$$

where ( $\mathrm{a}$ ) and ( $\mathrm{f}$ ) represent the aqueous and fused salt systems respectively. The notations and units used in this paper are summarized below*1. As shown in the equation 2, we assumed that the activity of the nitrate ion in the fused salt mixture is independent of the composition. From the equation of continuity,

$$
\begin{aligned}
\left(\partial C_{\mathrm{i}} / \partial t\right) & =-\left(\partial J_{\mathrm{i}} / \partial \mathrm{X}\right) \\
\mathrm{i} & =\mathrm{Na}^{+}, \mathrm{Cl}^{-} \\
\mathrm{i} & =\mathrm{Ag}^{+}, \mathrm{K}^{+}, \mathrm{NO}_{3}^{-} \text {( } \quad \text { ( f ) }
\end{aligned}
$$

On the other hand, the charge density due to the separation of charge is

$$
\begin{array}{ll}
\rho=F\left(C_{\mathrm{Na}^{+}}-C_{\mathrm{Cl}^{-}}\right) & \text {(a) } \\
\rho=F\left(C_{\mathrm{Ag}^{+}}+C_{\mathrm{K}^{+}}-C_{\mathrm{NO}_{3}}\right) & \text { (f) } \\
\text { Poisson's equation is } & \\
\partial^{2} \phi / \partial X^{2}=-(\rho / \varepsilon) \times 10^{2} & (\mathrm{a}, \mathrm{f})^{* 2}
\end{array}
$$

and Poisson's equation is

\subsection{Calculations}

The integration of Poisson's equation 6 was simplified according to the method described by Hafemann ${ }^{2}$. All of the charge in cell $\mathrm{j}$ is considered to lie on a

*1 Notations and units; $J=$ flux $\left(g-i o n / \mathrm{cm}^{2} \cdot \mathrm{sec}\right), D=$ diffusion coefficient $\left(\mathrm{cm}^{2} / \mathrm{sec}\right), C=$ concentration $\left(\mathrm{g}\right.$-ion $\left./ \mathrm{cm}^{3}\right), Y=$ activity coefficient, $U=$ mobility $\left(\mathrm{cm}^{2} / \mathrm{sec} \cdot \mathrm{V}\right), F=$ Faraday constant (coulomb), $\sigma=$ surface charge density $\left(\mathrm{c} / \mathrm{cm}^{2}\right), \rho=$ charge density $\left(\mathrm{c} / \mathrm{cm}^{3}\right), \varepsilon=$ absolute dielectric constant (Farad $\left./ \mathrm{m}\right)$, $\varepsilon^{\prime}=$ specific dielectric constant, $E=$ electric field intensity $(\mathrm{V} / \mathrm{cm}), \phi=$ potential $(\mathrm{V}), V=\operatorname{ditfusion}$ potential $(\mathrm{V}), \boldsymbol{X}=$ distance from the contact plane $(\mathrm{cm}), t=$ time $(\mathrm{sec}), d=$ cell thickness (cm)

-*2 MKS unit is used in the calculation. The factor $10^{2}$ is derived from reducing the unit of $\partial^{2} \phi / \partial X^{2}$ to $\mathrm{V} / \mathrm{cm}^{2}$ because $\varepsilon$ is given in Farad/m. plane at the middle point of the cell as a surface charge $\sigma_{j}$ (see Fig. 1).

$$
\sigma_{\mathbf{j}}=\rho_{\mathbf{j}} d
$$

where $d$ is the constant cell thickness. From the neutrality of the system as a whole, it follows

$$
\sum_{\mathbf{j}=0}^{\mathrm{m}} \sigma_{\mathbf{j}}=0
$$

By application of Gauss' theorem, the electric field intensity $E$ at $X\left(X_{\mathrm{k}} \leqslant X \leqslant X_{\mathrm{k}+1}\right)$ will be

$$
\begin{aligned}
E=-\partial \phi / \partial X & \simeq(1 / \hat{\varepsilon}) \times 10^{2} \times \sum_{\mathbf{j}=0}^{\mathbf{k}} \sigma_{\mathbf{j}} \\
& =(d / \mathrm{s}) \times 10^{2} \times \sum_{\mathbf{j}=0}^{\mathbf{k}} \rho_{\mathbf{j}}
\end{aligned}
$$

The potential at $X$ is given as

$$
\begin{aligned}
V= & -\int_{-\delta}^{X} E d X \simeq\left(d^{2} / \varepsilon\right) \times 10^{2} \\
& \times\left[\sum_{j=0}^{\mathrm{k}} \mathbf{j} \rho_{\mathbf{j}}-(\mathrm{k}+1) \sum_{\mathbf{j}=0}^{\mathrm{k}} \rho_{\mathbf{j}}\right] \\
= & \left(d^{2} / \varepsilon\right) \times 10^{2} \times \sum_{\mathbf{j}=0}^{\mathrm{k}} \mathbf{j} \rho_{\mathbf{j}}-(\mathrm{k}+1) E d
\end{aligned}
$$

where $\varepsilon$ is assumed to be constant.

The boundary conditions used in the calculation are as follows ( $\infty$ denotes the bulk of the solution where there exists no effect of the diffusion).

$$
\begin{aligned}
& C_{\mathrm{i}}(X, 0)=C_{\mathrm{i}}(-\infty, t) \quad-\delta \leqslant X<0 \\
& =C_{\mathrm{i}}(+\infty, t) \quad 0 \leq X<+\delta \\
& \left.C_{\mathbf{i}}(-\delta, t)=C_{\mathbf{i}}(-\infty, t)\right\} \\
& \left.C_{\mathbf{i}}(+\delta, t)=C_{\mathbf{i}}(+\infty, t)\right\} \\
& E(-\delta, t)=0 \\
& V(-\delta, t)=0 \\
& J_{\mathrm{i}}(-\delta, t)=0 \\
& \partial J_{\mathbf{i}} / \partial X=0 \quad \text { at } X= \pm \delta
\end{aligned}
$$

As shown in equations 13 and 14 the zero point for the electric field intensity and the potential was chosen at $X=-\delta$, and these conditions have been used in the calculations of equations 9 and 10 .

At time 0 the concentration of each cell is set to the initial conditions of the experiment being simulated, and values of $\partial C_{\mathbf{i}} / \partial X$ and $\partial \ln Y_{\mathbf{i}} / \partial X$ for every species $\mathrm{i}$ and $\partial \phi / \partial X$ are determined at the intersections of adjacent cells. Then the flux $J_{\mathfrak{i}}$ are calculated from 1 or 2 . These fluxes are assumed to remain constant over an appropriately short time interval $\Delta t$, and a new set of values for the concentrations is determined. From these values $\partial C_{i} / \partial X, \partial \ln Y_{\mathbf{i}} / \partial X$ and $\partial \phi / \partial X$ are again calculated, and the process is repeated as often as desired. In the calculation for fused salt $m+1=60$ and 100 repetition was used, and for aqueous solution $\mathrm{m}+1=100$ and 200 repetition was applied. It should be noted that the calculations can not yield true results after the time when the diffusion reaches either $X=-\delta$ or $+\delta$, because we treat here a free diffusion model. The machine 
used is NEAC-3100 or HITAC-5020 (double precision).

\subsection{Data used in this work}

2.5.1 $\mathrm{AgNO}_{3}-\mathrm{KNO}_{3}$ fused salt system The concentrations were calculated from the density data ${ }^{3)}$. As the external mobilites of the constituent ions at the temperature have not been measured, they were estimated as follows. The external mobility of the nitrate ion at $300^{\circ} \mathrm{C}$ was calculated from the value at $350^{\circ} \mathrm{C}^{4}$ with the estimated activation energy $3800 \mathrm{cal} /$ $\mathrm{g}$-ion of the nitrate ion in fused alkali nitrates ${ }^{5}$. The external mobilities of silver- and potassium ions were then calculable from the internal mobilities ${ }^{1)}$ and the estimated external mobility of the nitrate ion. The diffusion coefficients to be used here should be the mutual diffusion coefficients of ions without any effect of the local electric field and thus are experimentally unmeasurable. The values were then calculated from the mobilities by using Nernst-Einstein's relation $\left\{D_{\mathbf{i}}\right.$ $\left.=\alpha\left(R T U_{\mathrm{i}} / F\right)\right\}$. The $N-E$ factor $(\alpha)$ was varied in the calculations. The activity coefficients of silverand potassium ions calculated from the emf's of concentration cells ${ }^{6)}$ without the diffusion potentials ${ }^{1)}$ were reduced to the values corresponding to the concentration unit used here. The activity of the nitrate ion was assumed to be constant. The specific dielectric constant of the mixture was assumed to be constant with a mean value (1.485) of silver- and potassium nitrate ${ }^{7)}$.

2.5.2 NaCl aqueous system The mobilities were calculated from the equivalent conductances and transport numbers ${ }^{8)}$. The diffusion coefficients were obtained by using $N-E$ relation with the assumption

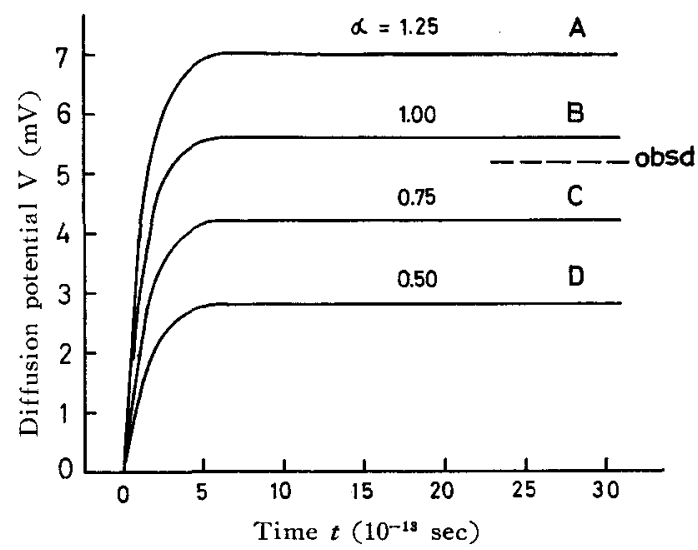

$\mathrm{AgNO}_{3}\left(0.5\right.$ m.f.), $\mathrm{KNO}_{8} / / \mathrm{AgNO}_{3}(0.3), \mathrm{KNO}_{3}$ at $300^{\circ} \mathrm{C}$. For all curves $\varepsilon^{\prime}=1.485, \Delta t=10^{-13} \mathrm{sec}, d=10^{-7} \mathrm{~cm}$

Fig. 2 Effect of Nernst-Einstein factor $(\alpha)$ on the diffusion potential vs. time curve for the fused-salt junction $\alpha=1$. The activity coefficients of sodium-and chloride ions were considered as the mean activity coefficients of sodium chloride ${ }^{8}$. The specific dielectric constant of the pure water $(78.54)^{8)}$ was used.

The functions obtained for the concentration range in question are listed below ${ }^{* 3}$.

\section{Results and Discussion}

Fig. 2, for the fused salt system, shows the variation of the potential with time across the diffusion layer when the various $N-E$ factors ( $\alpha$ ) were used. From the relation between the static potentials $\left(V_{\infty}\right.$ 's ; potentials at $t=\infty$ ) and $\alpha$ 's shown in Fig. 3 , the $\alpha$ corresponding to the observed diffusion potential (5.18. $\mathrm{mV}$ ) was found to be 0.925 for the silver- and potassium ions. Such a value so close to unity can suggest the identity of the species both for the diffusion and migration processes. Since a diffusion potential could

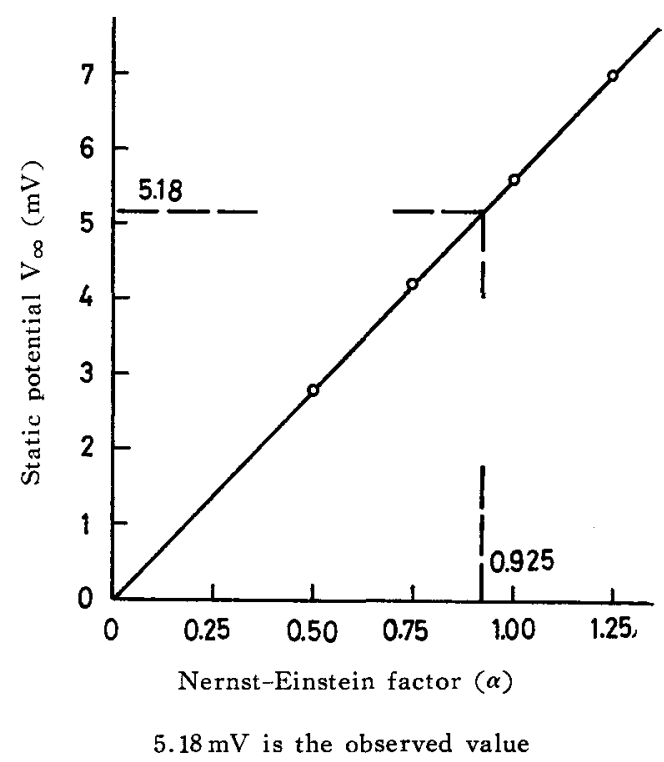

Fig. 3 Relation between the static diffusion potential and Nernst-Einstein factor for the fused-salt junction

\footnotetext{
*3 Fused salt system $\left(1=\mathrm{Ag}^{+}, 2=\mathrm{K}^{+}, 3=\mathrm{NO}_{3}{ }^{-}\right)$:

Mobility

$U_{1}=1.0 \times 10^{-2} \times C_{1}+2.32 \times 10^{-4}$

$U_{2}=1.75 \times 10^{-4}$

$U_{3}=-1.20 \times 10^{-4}$

Activity coefficient

$\ln Y_{1}=4.795 \times 10^{3} \times C_{1}{ }^{2}-5.274 \times 10 \times C_{1}-0.2188$

ln $Y_{2}=-5.481 \times 10^{3} \times C_{2}{ }^{2}+1.760 \times 10^{2} \times C_{2}-1.278$

Aqueous system $\left(1=\mathrm{Na}^{+}, 2=\mathrm{Cl}^{-}\right)$:

Mobility

$U_{1}=-4.02 \times 10^{-4} \times C_{1}+4.664 \times 10^{-4}$

$U_{2}=-4.94 \times 10^{-4} \times C_{2}+7.293 \times 10^{-4}$

Activity coefficient

$\ln Y_{1}=\ln Y_{2}=-1.068 \times C_{1}-1.426 \times 10^{-1}$
} 
not generate unless any diffusion occurs, it is reasonable that the extrapolated line crosses the origin as shown.

As shown in Fig. 4, the effect of the dielectric constant on the potential-time curve was examined for the fused salt system with hypothetical $\varepsilon^{\prime}$ s $(10,50$ and 100) while $\alpha$ was kept constant. The result shows that the relaxation time depends remarkably on the dielectric constant. As for the aqueous system in Fig. 5, the relaxation time is considerably longer than that in the fused salt system. This may be interpreted as a result of a larger dielectric constant of the aqueous solution than that of the fused salt (78.54: 1.485).

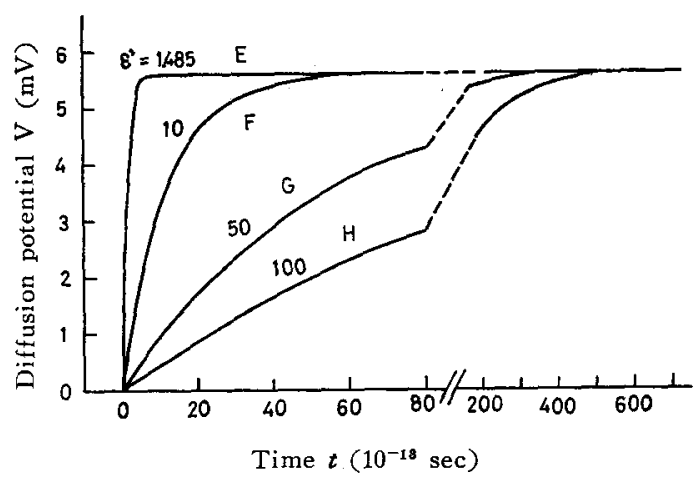

$\Delta t=10^{-18} \mathrm{sec}, d=10^{-7} \mathrm{~cm}$ for $E$ and $F$ $\Delta t=10^{-12} \mathrm{sec}, d=10^{-6} \mathrm{~cm}$ for $G$ and $H$ $\alpha=1.00$ for all curves

Fig. 4 Effect of dielectric constant on the diffusion potential vs. time curve for the fused-salt junction

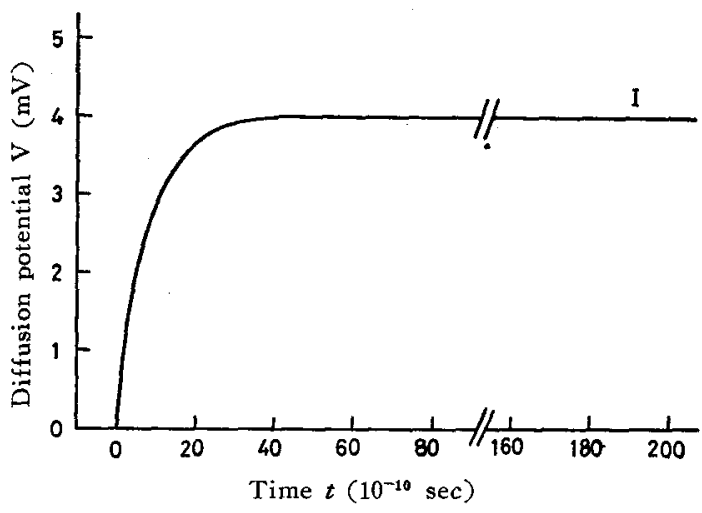

$\varepsilon^{\prime}=78.54, \alpha=1.00, \Delta t=10^{-10} \mathrm{sec}, d=10^{-7} \mathrm{~cm}$

Fig. 5 Diffusion potential vs. time curve for the junction $\mathrm{NaCl}(0.05 M) / / \mathrm{NaCl}(0.1 M)$ in an aqueous solution at $25^{\circ} \mathrm{C}$
Inorder to understand more thoroughly the variation of potential with time, we made here an attempt to obtain the relation between the ratio of potential relative to its steady value $\left(V / V_{\infty}\right)$ and the elapsing time as shown in Fig. 6. The line from $A$ to $I$ corresponds to each curve in Fig. 2, 4 and 5 . We can thus write the potential approximately as

$$
V=V_{\infty}\{1-\exp (-\beta t)\}
$$

And it seems that the diffusion coefficient gives no effect on the relaxation time, that is, $\beta$ is not a function of the diffusion coefficient, because the curves from $A$ to $D$ in Fig. 2 whose diffusion coefficients are varied by the various $\alpha$ values fit to the same line in Fig. 6. In Table 1, furthermore, we listed the time constant $(\tau=1 / \beta)$, that is the time when the potential equals

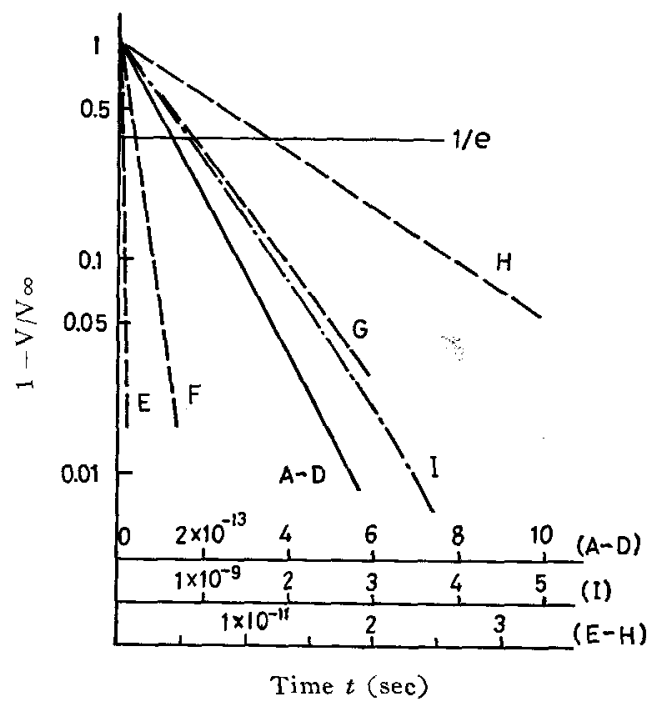

(A)-(I) represent the corresponding curves in Fig. 2, 4 and 5

Fig. 6 Relation between the diffusion potential relative to its steady value and the elapsing time

Table 1 Time constants

\begin{tabular}{c|c|c|c|c}
\hline & $\begin{array}{c}N-E \\
\text { factor }\end{array}$ & $\begin{array}{c}\text { Specific } \\
\text { dielectric } \\
\text { constant }\end{array}$ & $\begin{array}{c}\text { Time constant } \\
(\mathrm{sec})\end{array}$ & Curve \\
\hline \multirow{5}{*}{ Fused } & 1.25 & 1.485 & $1.2 \times 10^{-18}$ & $A$ \\
1.00 & 1.485 & $1.2 \times 10^{-18}$ & $B$ \\
& 0.75 & 1.485 & $1.2 \times 10^{-13}$ & $C$ \\
& 0.50 & 1.485 & $1.2 \times 10^{-13}$ & $D$ \\
\hline 1.00 & 1.485 & $1.2 \times 10^{-19}$ & $E$ \\
& 1.00 & 10 & $1.1 \times 10^{-12}$ & $F$ \\
\hline Aqueous & 1.00 & 50 & $5.5 \times 10^{-11}$ & $G$ \\
\hline
\end{tabular}


to $V_{\infty}(1-\exp (-1))$, calculated from Fig. 6 . It is concluded from the table that $\tau$ is roughly proportional to $\varepsilon$ and equation 17 can be written as

$$
V=V_{\infty}(D)\{1-\exp (-r t / \varepsilon)\}
$$

where $r$ is not a function of the diffusion coefficient. The qualitative explanation of equation 18 may be given as follows: The larger $\varepsilon$ yields the weaker electrostatic force which counteracts the diffusional migration of each constituent ion and thus brings about the longer relaxation time.

Since an analytical solution for equations from 1 to 6 has not been obtained, one can consider equation 18 as an approximate solution by a numerical calculation*4.

Figs. 7 and 8 show the charge separations for the fused salt and aqueous systems at time relative to $\tau$. The corresponding $V / V_{\infty}$ values were calculated from equation 18. The remarkable difference is observed between the two systems. The separation of charge

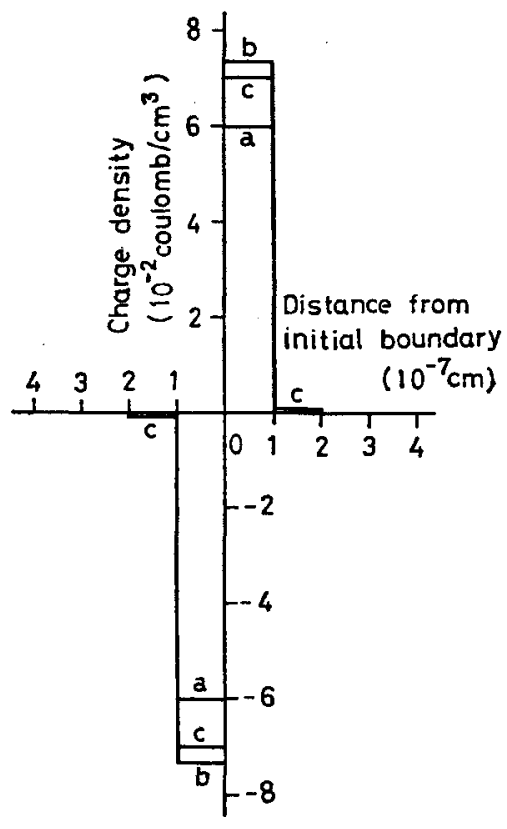

\begin{tabular}{c|c|c}
\multicolumn{3}{c}{$\tau=1.2 \times 10^{-18} \mathrm{sec}$} \\
\hline $\mathrm{a}$ & $\tau$ & $\mathrm{V} / \mathrm{V} \infty$ \\
\hline $\mathrm{b}$ & 1.54 & 0.7856 \\
$\mathrm{c}$ & 6.92 & 0.9990 \\
60.77 & $\approx 1$
\end{tabular}

$\varepsilon^{\prime}=1.485, \alpha=1.00, J_{t}=10^{-10} \mathrm{sec}, d=10^{-7} \mathrm{~cm}$

Fig. 7 Excess charge profile in the fused-salt diffusion layer

* The present authors found the work by Hickman ${ }^{97}$ during the revision of this manuscript. He has analytically solved the equations which are essentially same as those from 1 to 6 in this paper by means of perturbation techniques. in the fused salt can almost remain at such a long elapsing time relative to $\tau$ as $60.77 \tau$ while that in the aqueous solution can diffuse with time and close to neutrality even at $26.53 \tau$. A possible interpretation on fused salt system is given as follows: Since all the constituent ions can flow by the corresponding gradients of electrochemical potentials, a certain extent of the separation of charge could remain owing to the balance between the flows caused by the gradient of chemical potential and electrical field even after the steady state potential is attained. The remains of the separation of charge may suggest that generally-used condition of the neutrality throughout the diffusion layer in a solution of electrolytes does not hold in such a highly concentrated fused salt mixture composed of more than three ionic species as considered here. In the case of a fused salt, therefore, the diffusion between the two solutions which consist of a common solute and a predominant solvent will yield the results similar to those in an aqueous solution.

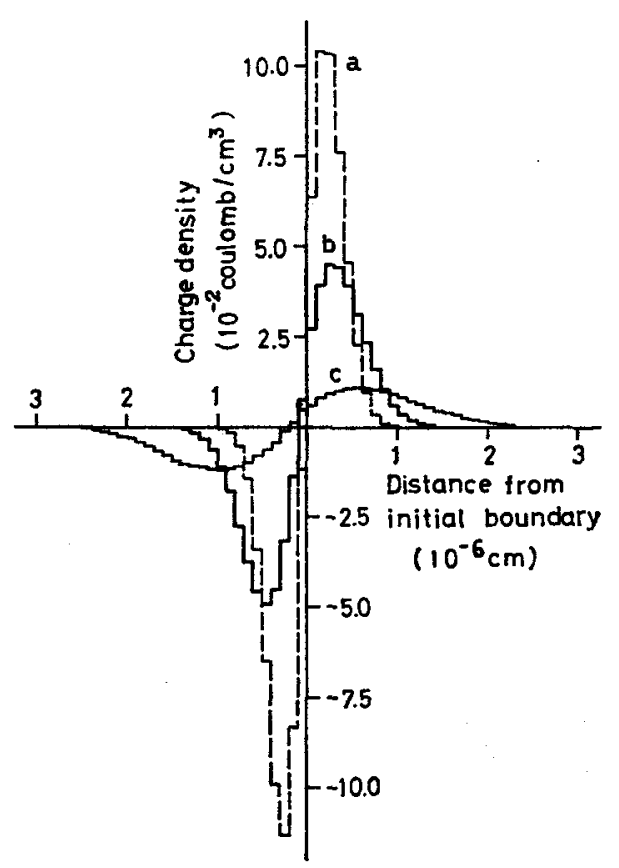

\begin{tabular}{c|c|c}
\multicolumn{3}{c}{$\tau=7.5 \times 10^{-10} \mathrm{sec}$} \\
\hline $\mathrm{a}$ & $\tau$ & $V N \infty$ \\
\hline $\mathrm{b}$ & 2.53 & 0.9204 \\
$\mathrm{c}$ & 6.53 & 0.9985 \\
& 26.53 & $\approx 1$ \\
\hline
\end{tabular}

Conditions in calculation are shown in Fig. 5

Fig. 8 Excess charge profile in the aqueous diffusion layer 
Distance from initial boundary $\left(10^{-6} \mathrm{~cm}\right)$
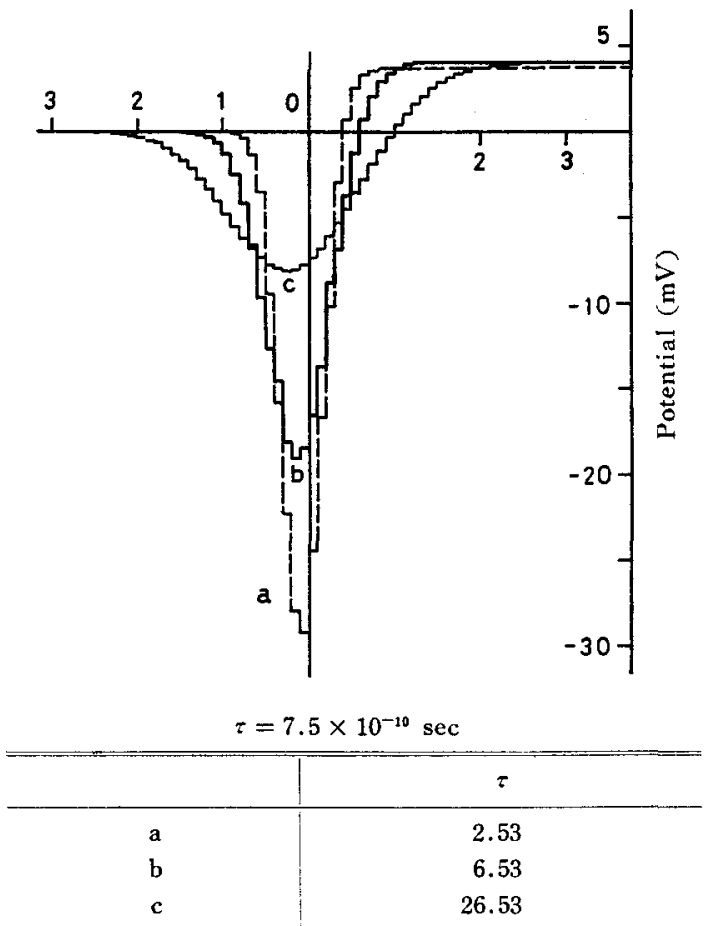

Conditions in calculation are shown in Fig. 5

Fig. 9 Potential profile in the aqueous diffusion layer
In the aqueous solution, the potential profile in the diffusion layer was calculated by equation 10 . As shown in Fig. 9, the minimum in the potential is observed near the contact plane.

Finally it should be mentioned that the phenomenological treatment in this work based on equations from 1 to 6 is somewhat macroscopic, the results will not make any physical sense at least before $\tau$. A more physical approach to the transition state problems could be made by microscopic statistical treatments based on interionic potentials including the effects of ionic sizes and local dielectric constants etc.

Acknowledgement: The authors wish to express their thanks to Dr. N.Sano, Departmemt of Metallurgy, University of Tokyo, for his suggestion in calculations.

\section{References:}

1) M. Okada, K. Kawamura, Electrochim. Acta 15, 1(1970).

2) D.R. Hafemann, J. Phys. Chem. 69, 4426 (1965).

3) F.R. Duke, R.A. Fleming, J. Electrochem. Soc. 103, 412 (1956).

4) F.R. Duke, B. Owens, ibid. 105, 476 (1958).

5) F. Lantelme, M. Chemla, Electrochim. Acta 11, 1023 (1966).

6) M. Bakes, J. Guion, Compt. Rend. 258, 1223 (1964).

7) G.J. Janz, "Molten Salts Handbook", p. 356 (1967), Academic Press, N. Y.

8) The Electrochemical Society of Japan, ed., "Denki-Kagaku Binran", p. 116, 118, 120,34 (1964), Maruzen, Tokyo.

9) H.J.Hickman, Chem. Eng. Sci. 25,381 (1970). 